\title{
Abdominelle Notfallsonografie in der Intensiv- und Akutmedizin
}

Michael Schreiber, Clemens-Alexander Greim

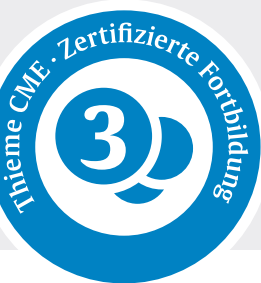

Die sonografischen Untersuchungstechniken unterstützen die Point-of-Care-Diagnostik (POC-Diagnostik) bei akutmedizinischen Fragestellungen. Für den Intensiv- und Notfallmediziner gilt: Kenntnisse und Fertigkeiten einer fokussierten Sonografie sind unabdingbar. Dieser Artikel beleuchtet die Optionen der abdominellen Notfallsonografie und gibt praktische Hilfestellung.

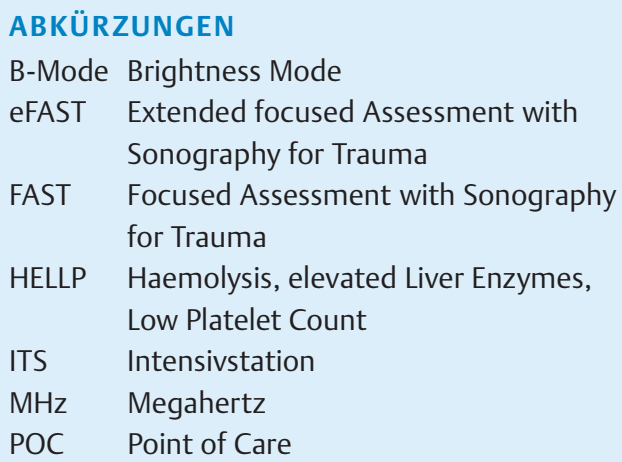

\section{Aktueller Stand}

Zunehmend kommen in der Akutmedizin kompakte und qualitativ hochwertige mobile Ultraschallgeräte bettseitig zum Einsatz [1, 2]. Neben der Unterstützung bei der Anlage zentralvenöser Katheter bieten die sonografischen Untersuchungen vor allem Hilfe bei der Beantwortung komplexer und therapeutisch relevanter klinischer Fragestellungen. Dabei sind es nicht die detaillierten Organbefunde, die zum schnellen und akuten Therapieerfolg beitragen, sondern häufig einfache Blickdiagnosen. Daher bieten die DGAI und die DEGUM (Deutsche Gesellschaft für Ultraschall in der Medizin) bereits seit Jahren verschiedene Sonografiekurse an, in denen auch die abdominelle Notfallsonografie abgebildet ist $[3,4]$.

Merke

Außerhalb der Regelarbeitszeit sind Sonografieexperten bei akutmedizinischen Fragen nicht immer rasch verfügbar. Dann helfen eigene Fähigkeiten in der POC-Sonografie dem Intensivmediziner bei schnellen, problemorientierten Therapieentscheidungen [3].

Auf der Intensivstation oder in Notfallsituationen sind Anästhesisten regelmäßig mit typischen klinischen Problemen aus dem Bereich des Abdomens konfrontiert:
- Blutungen und hämodynamische Instabilität

- pathologisch veränderte Gallenwege und Störungen der Magen-Darm-Passage

- akutes Nierenversagen und/oder Störungen der Harnableitung

Die Sonografie kann zahlreiche der damit verbundenen Fragestellungen beantworten. Dafür benötigt der Untersucher eine gute technische Ausstattung.

\section{Praktisches Vorgehen}

Praktischerweise verfügen heutzutage bereits viele Anästhesisten und Intensivmediziner über sonografische Erfahrungen im Rahmen von Regionalanästhesie, Thoraxund/oder Kardiosonografie. Im Folgenden werden darauf aufbauend die richtige Schallkopfwahl, Methoden zur Darstellungsoptimierung und Bilddokumentation für das Feld der Abdomensonografie vorgestellt. Im Weiteren wird als Grundlage das FAST-Konzept (Focused Assessment with Sonography for Trauma) dargestellt, von dessen Standardschnitten durch Modifikation weitere fokussierte Organinformationen abgeleitet werden können. Die fokussierte Abdomensonografie stellt aber nach wie vor einen konzeptionell jungen Bereich dar. Mögliche Qualifikationserfordernisse sind bereits in der Entwicklung und sollen abschließend skizziert werden.

\section{Wahl des Schallkopfes}

Die Sendefrequenz und die kurvige Anordnung der piezoelektrischen Kristalle des Konvexschallkopfes stellen einen Kompromiss dar zwischen

- ausreichender Schalleindringtiefe,

- sektorförmiger Erfassung von tiefgelegenen Strukturen auch in der Breite und

- relativ guter Nahauflösung. 
Merke

In der fokussierten Abdomensonografie liefert ein 3,5bis $5-\mathrm{MHz}-K o n v e x s c h a l l k o p f$ bei einer Eindringtiefe von ca. 15-20 cm die wichtigsten Informationen.

\section{Sonografischer Darstellungsmodus}

Am Ultraschallgerät ermöglichen zahlreiche Menüs eine optimale Bildeinstellung für die geplante Untersuchung. Die Geräte sind in der Regel vorkonfiguriert („presets“). Sie können durch den Untersucher mittels verschiedener Vorund Nachverarbeitungsoptionen (Gain, Fokussierung, Dynamic Range etc.) an die speziellen Erfordernisse angepasst werden. Für die wichtigsten abdominellen Untersuchungsgänge wird meist nur der Brightness Mode (B-Mode) benötigt, der das klassische sonografische Bild liefert ( $\triangleright$ Abb. 1).

Als wichtige Konvention der Abdomensonografie gilt: Kraniale Strukturen im Sagittalschnitt sowie die rechte Patientenseite im Transversalschnitt sind auf dem Monitor jeweils links randbildend. Bei der Bilddokumentation wird diese Absprache durch ein sog. Piktogramm und die jeweiligen Patientendaten ergänzt.

\section{Untersuchungskonzepte und Schnittebenen}

Das FAST-Konzept ist die wohl am weitesten verbreitete und am besten evaluierte sonografische Untersuchungsmethode für fokussierte klinische Fragestellungen ( $\triangleright$ Tab. 1). Ursprünglich wurde FAST für die thorakoabdominelle Sofortdiagnostik bei polytraumatisierten Patienten entwickelt [5]. Nach heutigem Stand wird FAST in 5 standardisierten Schnitten dazu verwendet, nach freier Flüssigkeit in den Pleuraräumen und tiefen peritonealen Umschlagsfalten zu fahnden ( $\triangleright$ Abb. 2). Manche Autoren ergänzen den Untersuchungsgang durch eine 6. Position, den subkostalen Vierkammerblick der transthorakalen Echokardiografie [6]. Vorteile werden im schnellen Nachweis eines Perikardergusses, der Beurteilung der kardialen Füllung sowie in einer orientierenden Einschätzung der Pumpfunktion gesehen. In jüngster Zeit wurden diese 6 Standardschnitte noch um die Pneumothoraxdiagnostik erweitert und unter dem Namen eFAST (extended FAST) publiziert [7].

Bei der Traumadiagnostik im Schockraum gehört die FAST-Untersuchung bereits nach ATLS ${ }^{\circledR}$ (Advanced Trauma Life Support) und ETC ${ }^{\circledR}$ (European Trauma Course) zum festen Algorithmus [8, 9]. Daneben hat sie auch auf der Intensivstation einen Stellenwert. Im Schockraum hilft sie in zeitkritischen Situationen und bei vitaler Instabilität bei der Detektion freier Flüssigkeit und als Entscheidungshilfe zur frühen Laparotomie [10]. Der positive FAST-Befund erreicht dabei eine ähnliche Sensitivität wie die CT [11]. Diese enthält darüber hinaus allerdings spezifischere Informationen zu Verletzungen, die die Sonografie nicht erbringen kann, ist aber $u$. U. mit höherem Zeitund Logistikaufwand verbunden.

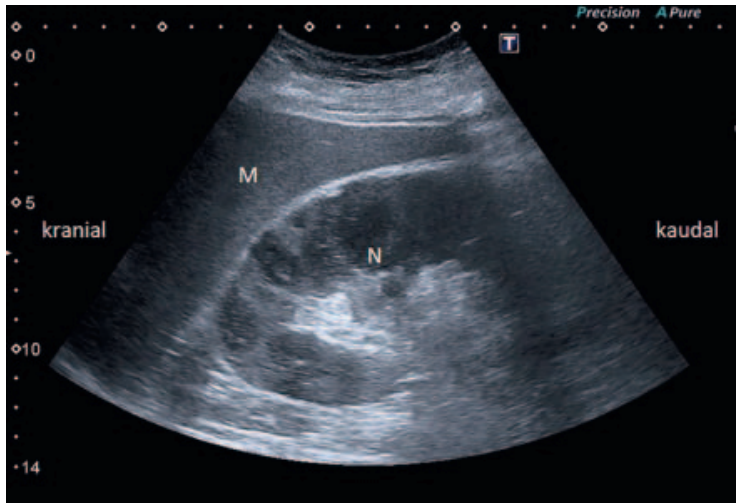

- Abb. 1 Sonogramm der Milz (M) im klassischen B-Bild eines 3,5- bis 5-MHz-Konvexschallkopfes. N: Niere. Quelle: Schreiber M, Greim CA. Akutsonografie in der Intensivmedizin: Abdomen. In: Eckart J, Forst $\mathrm{H}$, Briegel J, Hrsg. Intensivmedizin. Kapitel XV - 4.4. Landsberg: ecomed Medizin; 2018: 2.

Cave
Bei massiver Unfallkinematik und konsekutivem Verdacht auf umfangreiche Begleitverletzungen sowie Schockzeichen muss auch bei negativem FAST-Befund zwingend eine weiterführende CT erfolgen.

Auf der Intensivstation liegen die Indikationen für FAST/ eFAST in der Verlaufskontrolle nach abdominellem Trauma, gefäß- und viszeralchirurgischen Operationen sowie in der Pleuraerguss- und Aszitesdiagnostik.

Die FAST-Untersuchung ist - bei strukturierter Didaktik nachweislich an einem Tag zu erlernen [12]. Dabei wird das Plateau der Lernkurve bereits regelmäßig nach ca. 10 Untersuchungen erreicht.

Merke

Zum schnellen Erlernen und Reproduzieren der FASTUntersuchung soll deren Reihenfolge streng eingehalten werden. Durch diese Standardisierung können klinisch relevante Befunde innerhalb von wenigen Minuten erhoben werden.

\section{Klinische Fragestellungen}

\section{Blutungen und hämodynamische Instabilität}

Bei polytraumatisierten Patienten zählen Verletzungen der Leber, der Milz und der Nieren zu den häufigsten Ursachen für eine intraabdominelle Ansammlung freier Flüssigkeit. Im Thoraxbereich stehen meist Blutgefäßverletzungen und Lungeneinrisse hinter einem Hämatothorax. Daneben müssen bei Kreislaufinstabilität eine Perikardtamponade und ein Spannungspneumothorax ausgeschlossen werden. 
- Tab. 1 eFAST-Positionen „auf einen Blick“.

\begin{tabular}{|c|c|c|c|}
\hline Nr. & Fragestellung & Strukturen & Bezeichnung \\
\hline 1 & Pleuraerguss? & Leber und Lungenartefakt & rechter Pleura-Randwinkel \\
\hline 2 & Flüssigkeitssaum zwischen Leber und Niere? & Leber und rechte Niere & Morison-Pouch \\
\hline 3 & Pleuraerguss? & Milz und Lungenartefakt & linker Pleura-Randwinkel \\
\hline 4 & Flüssigkeitssaum zwischen Milz und Niere? & Milz und linke Niere & Koller-Pouch \\
\hline 5 & Blasenstatus, Flüssigkeit in Blasenumgebung? & Harnblase, ggf. Uterus & Douglas-Raum \\
\hline (6) & $\begin{array}{l}\text { Perikarderguss? } \\
\text { Volumenstatus? } \\
\text { Pumpfunktion? }\end{array}$ & Leber, Herz, Perikard & subkostaler Vierkammerblick \\
\hline (7) & Pneumothorax & $\begin{array}{l}\text { Pneumothorax-Algorithmus } \\
\text { (s. Veröffentlichungen zum Thema) }\end{array}$ & Bat Sign \\
\hline
\end{tabular}

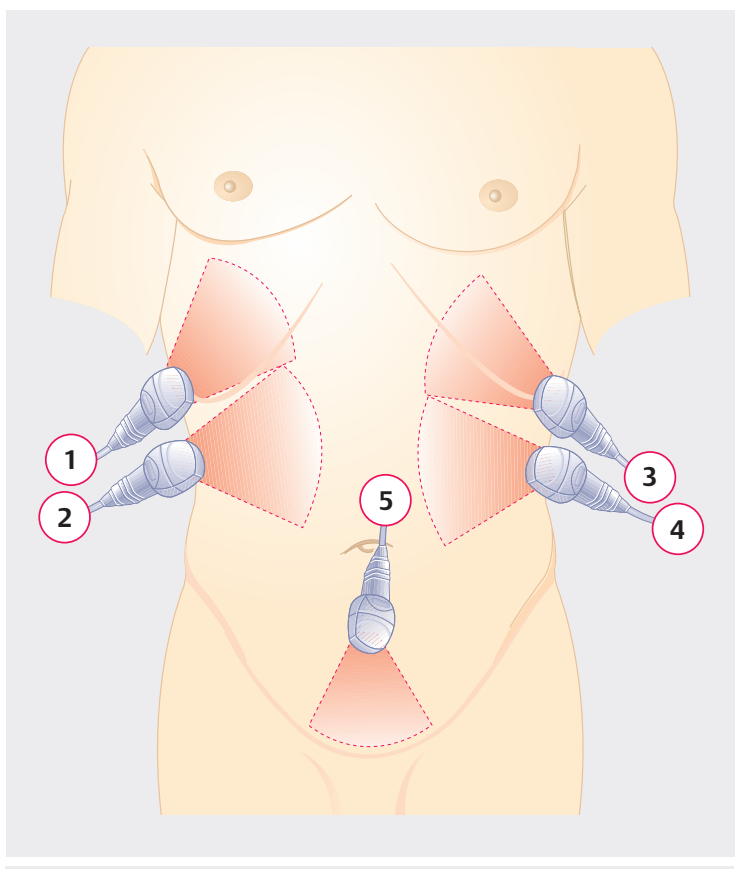

- Abb.2 Anlotungspunkte im klassischen FAST. 1: lateraldiaphragmaler Längsschnitt rechts: Pleuraraum, subphrenisch; 2: lateral-kaudaler Längsschnitt rechts: MorisonPouch, perihepatisch, Retroperitoneum; 3: lateraldiaphragmaler Längsschnitt links: Pleuraraum, subphrenisch, perisplenisch; 4: lateral-kaudaler Längsschnitt links: KollerPouch, Retroperitoneum; 5: medianer Unterbauchschnitt quer/längs: retro- und paravesikal. Quelle: Helm M, Hauke J, Lampl L. Apparative Diagnostik und Monitoring. In: Scholz J, Sefrin P, Böttiger B et al., Hrsg. Notfallmedizin. 3. Aufl. Stuttgart: Thieme; 2013: 86.

\section{Hämatothorax und Pleuraerguss}

Die FAST-Positionen 1 und 3 dienen zur Detektion freier Flüssigkeit in den Pleurahöhlen. Im posttraumatischen Setting wäre bei positivem Befund ein Hämatothorax wahrscheinlich. Beide Schnitte helfen regelmäßig auf der Intensivstation auch bei der Abklärung von Pleuraergüs- sen z. B. im Rahmen des Weaning-Versagens. Ein äußerst wichtiger praktischer Aspekt, gerade vor einer Punktion, ist die sonografische Identifizierung des Diaphragmas. Nur so lässt sich genau herausarbeiten, ob sich die freie Flüssigkeit tatsächlich im Pleuraraum oder um Leber bzw. Milz befindet. Bei negativem Befund legt sich in Inspiration die Lunge über Leber bzw. Milz.

- Abb. 3, 4 zeigen 2 verschiedene Ausprägungen von posttraumatischer freier Flüssigkeit. Sonomorphologisch stellt sich Flüssigkeit ohne Echos, also schwarz, dar. - Abb. 3 zeigt einen großen Erguss, der kompressiv auf die Lunge wirkt und sukzessive zur Atelektase führt. - Abb. 4 hingegen zeigt ein geringeres Trauma mit kleinerem Anteil freier Flüssigkeit (hier nach Rippenfraktur). Da bereits $20 \mathrm{ml}$ Flüssigkeit sonografisch detektierbar sind, hat die Sonografie somit eine bessere Sensitivität als die konventionelle Röntgendiagnostik. Der CT ist sie jedoch immer noch unterlegen.

\section{Merke}

Für die Punktionsplanung liegen die Vorteile der Sonografie in der Abschätzung des Flüssigkeitsvolumens, der Detektion solider oder gekammerter Prozesse und der sicheren Festlegung der Punktionsstelle.

\section{Perikardtamponade}

Unter physiologischen Bedingungen ist die Perikardhöhle nur mit ca. 10-15 ml Flüssigkeit gefüllt. Wegen der kollagenbedingten geringen Dehnbarkeit des Perikards haben größere Flüssigkeitszunahmen in der Perikardhöhle teils massive hämodynamische Auswirkungen. Von einer Perikardtamponade spricht man formal, wenn der Erguss die Füllung des Herzens mit Schockfolge behindert. Dies kann der Fall sein nach Thoraxtrauma oder kardiochirurgischen Eingriffen, aber auch bei schweren transmuralen Infarkten oder einer Stanford-A-Dissektion [13]. 


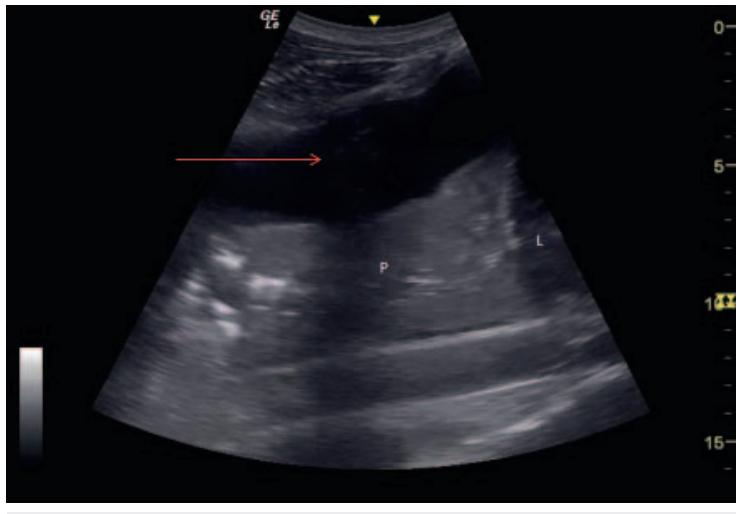

- Abb. 3 FAST-Position 1 mit positivem Befund. L: Leber, P: Lunge, Pfeil: hypoechogener Erguss. Quelle: Schreiber M, Greim CA. Akutsonografie in der Intensivmedizin: Abdomen. In: Eckart J, Forst H, Briegel J, Hrsg. Intensivmedizin. Kapitel XV - 4.4. Landsberg: ecomed Medizin; 2018: 4.

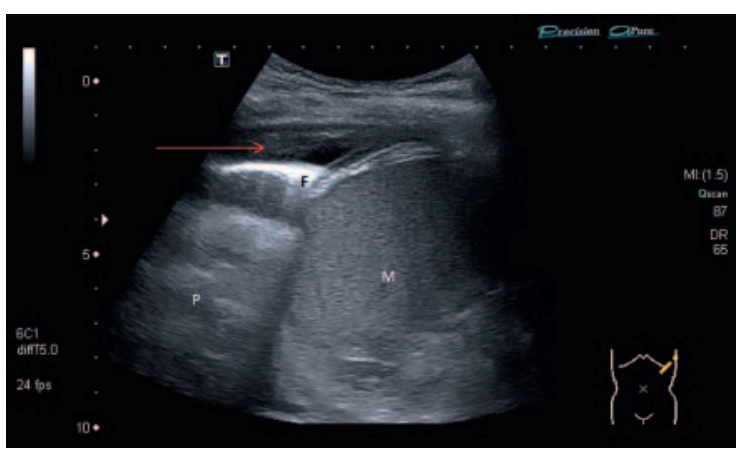

- Abb. 4 FAST-Position 3 mit positivem Befund. M: Milz, P: Lunge, F: Rippenfraktur, Pfeil: kleiner Erguss. Quelle: Schreiber M, Greim CA. Akutsonografie in der Intensivmedizin: Abdomen. In: Eckart J, Forst $\mathrm{H}$, Briegel J, Hrsg. Intensivmedizin. Kapitel XV - 4.4. Landsberg: ecomed Medizin; 2018: 4.

Bei einem hämodynamisch instabilen Patienten ist die Sonografie in der Akutsituation das Verfahren der Wahl, um eine Perikardtamponade auszuschließen. Eingestellt wird der subkostale Vierkammerblick, entsprechend der das FAST-Konzept ergänzenden Schallkopfposition 6. Dabei zeigt sich bei sukzessiver Druckzunahme im Perikard zunächst ein Kollaps des rechten Atriums (ggf. mit oberer Einflussstauung) und später des rechten Ventrikels. Im späten Stadium der Tamponade wird regelmäßig ein im Erguss „tanzendes“ Herz gesehen (Swinging Heart Sign).

Große Perikardergüsse mit einer Saumbreite von $>2 \mathrm{~cm}$, z. B. im Rahmen chronischer Erkrankungen, müssen nicht zwingend zu einer Tamponade führen. Andererseits kann, z. B. bei massiver Hypovolämie eines polytraumatisierten Patienten, ein Erguss mit einem zirkulären Saum $<1 \mathrm{~cm}$ eine bereits bestehende hämodynamische Einschränkung verstärken. Wie beim Pleuraerguss sollte

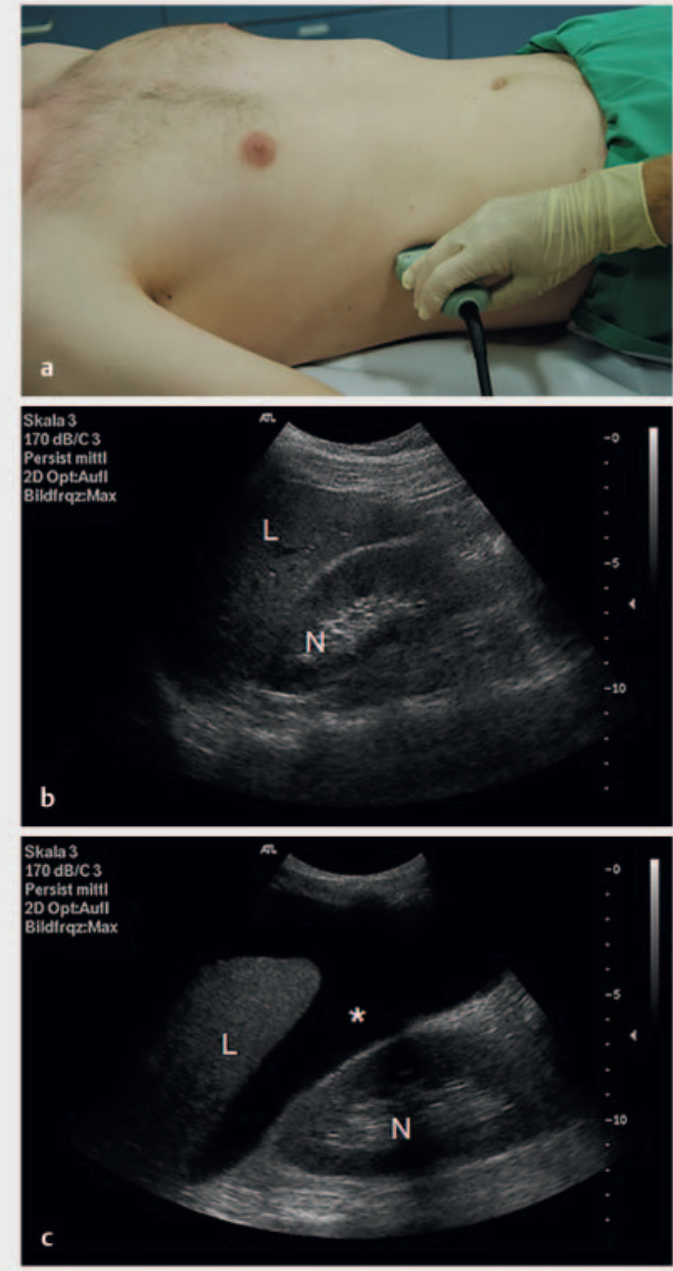

- Abb. 5 FAST-Position 2: Morison-Pouch. L: Leber, N: Niere. a Darstellung der Schallkopfführung. b Physiologischer Morison-Pouch. c Pathologischer Morison-Pouch mit Erguss $\left({ }^{*}\right)$. Quelle: Bord JP et al. Zielorientierte Notfallsonografie bei Traumapatienten (FAST-Protokoll). Dtsch Med Wochenschr 2008; 133: 2646-2648.

die Sonografie auch bei der Entlastung einer Perikardtamponade zur Steuerung der Ergusspunktion herangezogen werden.

\section{Merke}

Die Sonografie ist in der Notfallsituation das Verfahren der Wahl für den Ausschluss einer Perikardtamponade sowie bei der Entlastung einer Perikardtamponade zur Steuerung der Punktion.

\section{Leberruptur und -hämatom}

Bei abdominellen Traumata, aber auch bei Lebererkrankungen, z. B. HELLP (Haemolysis, elevated Liver Enzymes, low Platelet Count) oder Tumoren kann es zu Leberrupturen mit Hämatombildung und hämodynamischer Kompromittierung kommen. 
Die FAST-Position 2 eignet sich zur orientierenden Darstellung des rechten Leberlappens und stellt den Raum zwischen kaudaler Leber und dem oberen Nierenpol dar ( $\triangleright$ Abb. 5). In diesem sog. Morison-Pouch kann sich nach einem abdominellen Trauma durch die Taschenbildung des Peritoneums freie Flüssigkeit ansammeln. Diese wird in 60-90\% der Fälle sonografisch erkannt [14, 15]. Auch nach gastroenterologischen oder endovaskulären Interventionen ist das Auftreten von Leberhämatomen beschrieben.

\section{FALLBEISPIEL}

\section{Rupturiertes Leberhämatom}

Die diensthabende Gynäkologin bittet um postpartale Überwachung einer 35-jährigen Patientin, die am Vortag eine Sectio bei Beckenendlage erhalten hat. Derteit habe sie massive Oberbauchschmerzen mit nicht einstellbarem arteriellem Hypertonus und somit bestehe der Verdacht auf schweres HELLP-Syndrom. Es erfolgt ein arztbegleiteter Transport vom 8. OG auf die Intensivstation (ITS) im Erdgeschoss. Dabei erleidet die Patientin im Aufzug einen GrandMal-Anfall. Bei Ankunft auf der ITS liegen A-D-Probleme vor, und es besteht Unklarheit über den instabilen Zustand der Patientin. Aufgrund der einsetzenden Schockzeichen wird eine FAST-Sonografie durchgeführt. Diese ergibt neben freier Flüssigkeit im Abdomen ein großes intrahepatisches Hämatom (wie in Abb.6). Die Patientin wird ohne weitere Bildgebung sofort der operativen Versorgung zugeführt, bei der ein rupturiertes intrahepatisches Hämatom ausgeräumt wird.

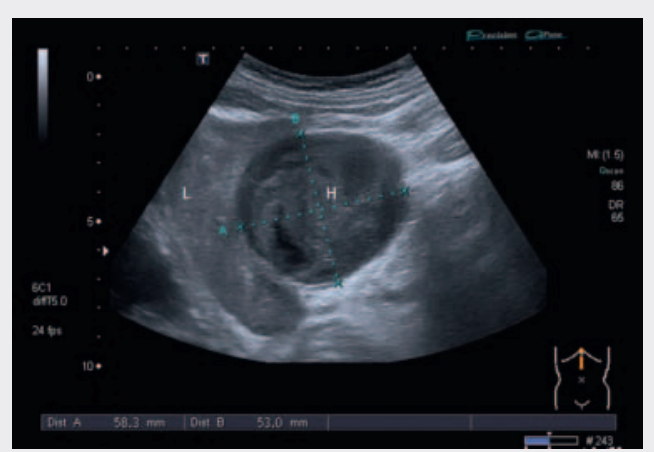

> Abb. 6 Leberhämatom mit Diameter A und B. L: Leber, H: Hämatom.

\section{Milzverletzungen}

Analog zur FAST-Position 2 zeigt die FAST-Position 4 auf der linken Körperseite den Raum zwischen oberem Nierenpol und Milz, den sog. Koller-Pouch ( $\boldsymbol{A}$ Abb. $\mathbf{7}$ ). Dort sammelt sich z. B. nach stumpfem Bauch- oder linksseiti-

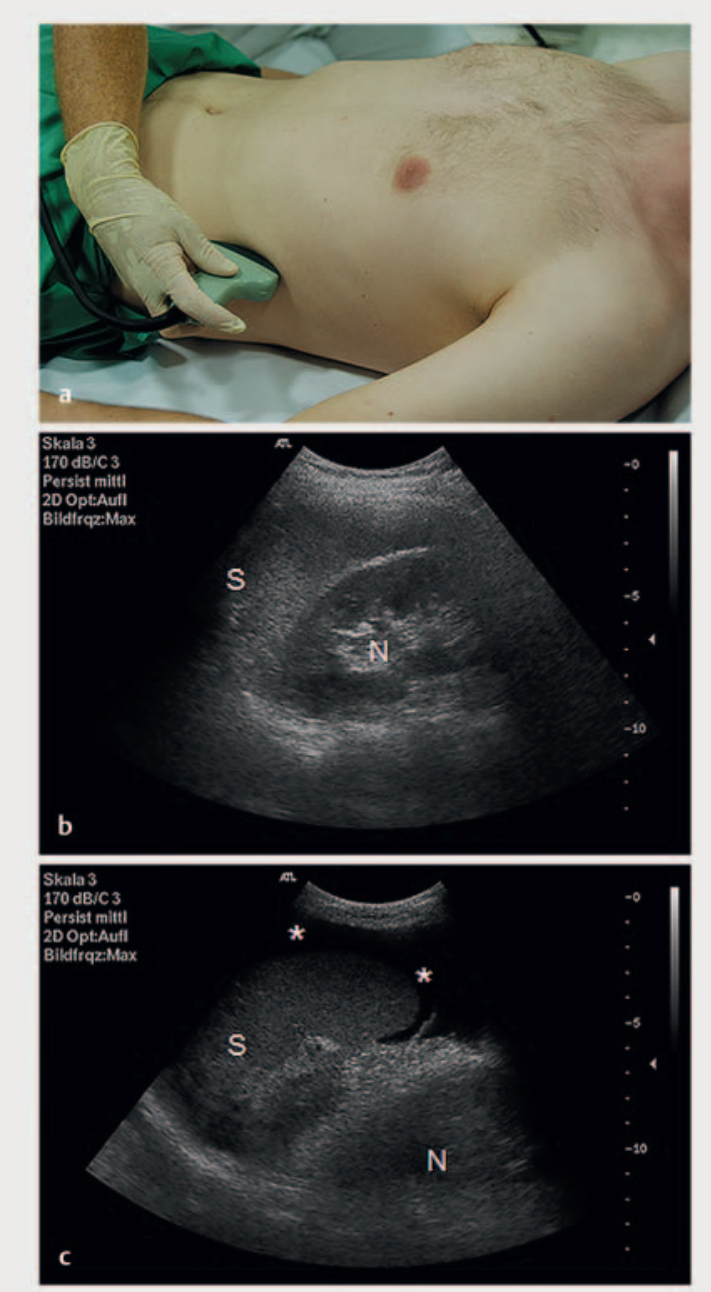

- Abb. 7 FAST-Position 4: Koller-Pouch. S: Milz, N: Niere. a Darstellung der Schallkopfführung. b Physiologischer Koller-Pouch. c Pathologischer Koller-Pouch mit Erguss ${ }^{*}$ ). Quelle: Bord JP et al. Zielorientierte Notfallsonografie bei Traumapatienten (FAST-Protokoll). Dtsch Med Wochenschr 2008; 133: 2646-2648.

gem Thoraxtrauma klassischerweise freie Flüssigkeit. Bei Verdacht auf eine primäre oder sekundäre Milzruptur ist der Befund eines perisplenischen Flüssigkeitssaums meist wegweisend. Da Niere und Milz etwas kranialer liegen als die Organe der Gegenseite, ist die Diagnostik wegen der durch die Rippen entstehenden sonografischen Schatten jedoch manchmal erschwert. Hinzu kommt, dass die Schallregion - je nach Füllung des Magens mit Luft und/ oder Flüssigkeit - überlagert sein kann.

\section{Merke}

FAST fahndet mit 6 standardisierten Positionen nach intraabdomineller freier Flüssigkeit, u. a. im Morison-, Koller-Pouch und Douglas-Raum. Nach Trauma ist die sonografische Verlaufskontrolle wichtig zur Frühdetektion einer zweizeitigen Milzruptur. 


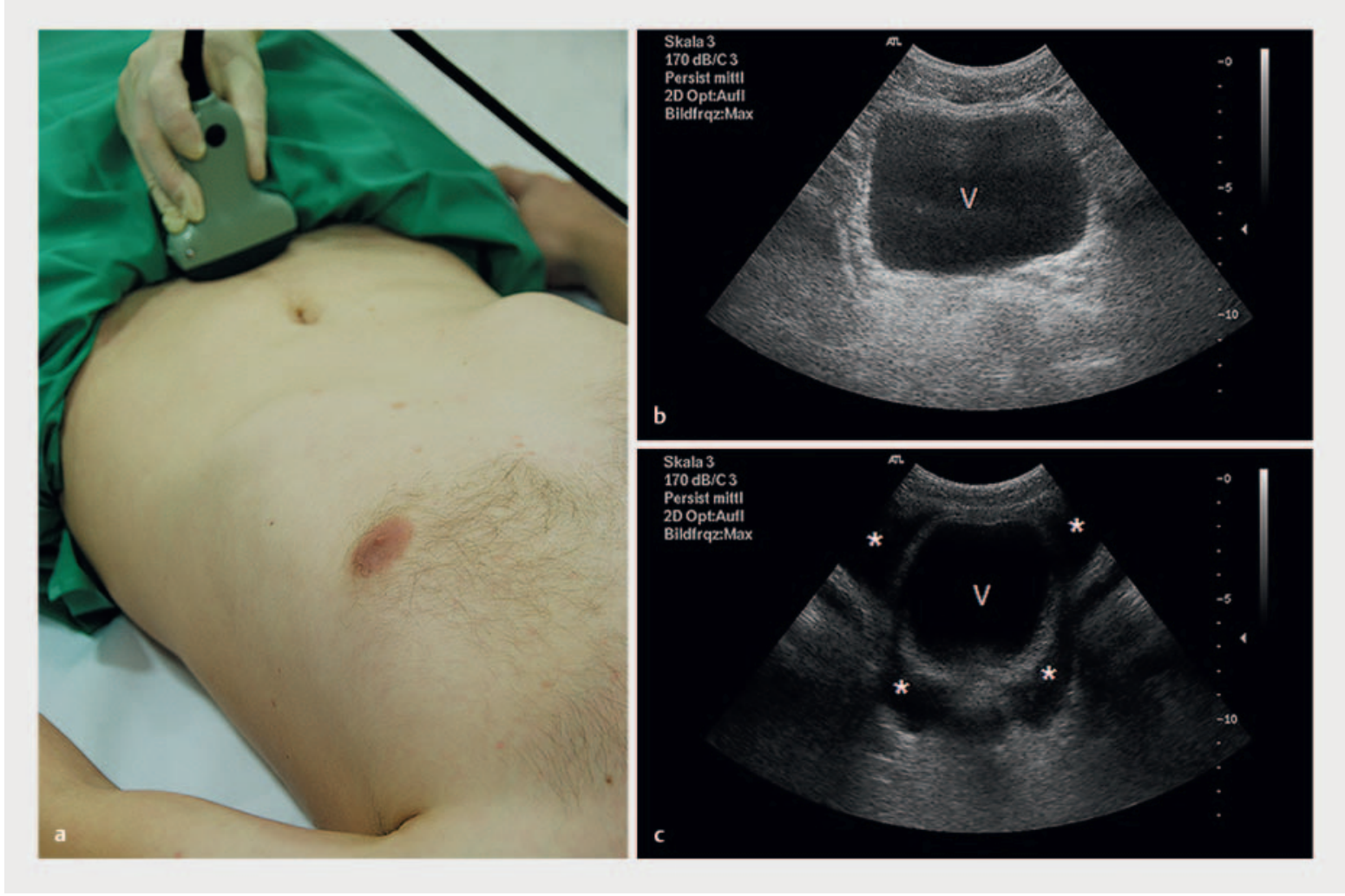

- Abb. 8 FAST-Position 5: Douglas-Pouch. V: Harnblase. a Darstellung der Schallkopfführung. b Physiologischer Douglas-Pouch. c Pathologischer Douglas-Pouch mit Erguss (*). Quelle: Bord JP et al. Zielorientierte Notfallsonografie bei Traumapatienten (FASTProtokoll). Dtsch Med Wochenschr 2008; 133: 2646-2648.

\section{Harnblasenruptur}

Vor allem bei Rasanztraumata ist auch die Harnblase sehr verletzungsgefährdet. Da Flüssigkeit physikalisch nicht komprimierbar ist, kann eine gefüllte Harnblase in dieser Situation leicht rupturieren. Darüber hinaus sammelt sich freie intraabdominelle Flüssigkeit der Schwerkraft folgend auch um die Harnblase.

Mit der FAST-Position 5 werden bei Frauen der perivesikale und der retrouterine Raum (Douglas-Pouch), bei Männern der rektovesikale Spalt sonografiert ( $\triangleright$ Abb. 8). Bei sehr viel freier intraabdomineller Flüssigkeit bilden die peritonealen Umschlagsfalten in diesen Regionen größere Flüssigkeitsreservoirs. Dort können sonografisch gelegentlich sogar schwimmende Darmschlingen differenziert werden (Anemonenphänomen).

\section{Cave}

Ein typischer Fallstrick ist hier die Verwechslung von Gefäßen oder flüssigkeitsgefülltem Darm, insbesondere dem Rektum, mit freier abdomineller Flüssigkeit.

Ein luftgefüllter Darm kann die Sicht einschränken, dagegen kann eine uringefüllte Blase die Orientierung in Position 5 sehr erleichtern. Je geringer das Blasenvolumen ist, desto schwieriger ist die Flüssigkeitsansammlung sono- grafisch nach intravesikal und perivesikal zu unterscheiden.

Großen Stellenwert haben die sonografischen Schnitte der Harnblase bei der Anlage eines suprapubischen Katheters. Sie ermöglichen die Punktion unter visueller Kontrolle und reduzieren das Komplikationsrisiko. Darüber hinaus lassen sich sonografisch auch Blutansammlungen im Sinne einer Blasentamponade diagnostizieren, die z. B. als postoperative Komplikation nach transurethraler Prostata- oder Blasenresektion entstehen.

\section{Akute Funktionsstörungen intraabdomineller und retroperitonealer Organe}

Bei intensivmedizinischen Krankheitsbildern bestehen zahlreiche Risikofaktoren für intra- und retroperitoneale Organinsuffizienzen:

- die Entzündungsmediation bei Sepsis oder Trauma,

- Nahrungskarenz,

- maschinelle Beatmung, aber auch

" die Verabreichung multipler Pharmaka.

Störungen der Gallendrainage und Magen-DarmPassage

Vor allem kreislaufbedingte Mikrozirkulationsstörungen, eine verlängerte Hypoxie und die Stase der Gallenflüssig- 
keit erhöhen das Risiko für die Ausbildung einer akalkulösen Cholezystitis. Dahinter stehen eine Entzündung der Gallenblasenwand sowie Motilitätsstörungen der Gallenwege, die klinisch auch als Schockgallenblase bezeichnet werden.

\section{Merke}

Die akalkulöse Cholezystitis ist eine seltene, aber schwerwiegende Erkrankung des Intensivpatienten, deren frühzeitige sonografische Detektion septische Komplikationen vermeiden hilft.

Die schnelle Diagnosestellung der akalkulösen Cholezystitis wird dadurch erschwert, dass die klassischen Zeichen - Murphy-Zeichen, Fieber, Übelkeit, Erbrechen, Abwehrspannung - oft durch Analgosedierung und Beatmung maskiert sind. Unter den ergänzenden apparativen Untersuchungen stellt die POC-Sonografie des Abdomens dann das Verfahren der ersten Wahl dar. Aufgrund des hohen klinischen Stellenwerts findet sich ihr frühzeitiger Einsatz auch als Empfehlung in der S2k-Leitlinie der deutschen Sepsis-Gesellschaft [16]. Die POC-Sonografie kann heute in vielen Fällen eine zeitnahe Cholezystektomie als entscheidende Therapiemaßnahme innerhalb von 6 Stunden nach Diagnosestellung bahnen [17].

\section{FALLBEISPIEL}

\section{Gallenblasenhydrops}

Ein 22-jähriger Patient nach Rollerunfall liegt den 15. Tag auf der Intensivstation. Im Sinne der DamageControl-Surgery sind die Beckenfraktur sowie die linksseitige Humerus- und Femurfraktur mit externen Fixateuren versorgt. Bei stattgehabter Lungenkontusion erfolgte bereits eine dilatative Frühtracheotomie. Bisher gelang aufgrund der Magen-Darm-Atonie noch keine enterale Vollernährung. Obwohl das Abdomen keinerlei Traumaschäden erlitt, kommt es seit Tagen zu einem Anstieg von Gamma-GT, AP und Transaminasen. Trotz des fehlenden akuten Infektionsverdachts halten sich die Entzündungszeichen auf leicht erhöhtem Niveau. Sedierungsbedingt ist die klinische Beurteilung des Abdomens eingeschränkt, ein positives Murphy-Zeichen aber nicht auszuschließen.

Es erfolgt eine fokussierte Sonografie der Gallenblase. Dabei stellt sich ein massiver Gallenblasenhydrops mit reaktiver Entzündung dar. Aufgrund des Befunds wird von der Viszeralchirurgie und der Radiologie in gemeinsamer Absprache die Indikation zur perkutanen transhepatischen Cholangiodrainage (PTCD) gestellt. Fortan normalisieren sich die Leber- und Entzündungszeichen. Auch der Kostaufbau gelingt immer besser.

Für die Sonografie der Gallenblase benötigt man den subkostalen Schrägschnitt, den verlängerten Interkostal- schnitt und manchmal den Interkostalschnitt. Im Ultraschall sind klassische Zeichen der Cholezystitis der Gallenblasenhydrops (Normalgröße $<100 \mathrm{~mm}$ ), die Wandverdickung (normale Wanddicke $<3 \mathrm{~mm}$ ) und ggf. freie Flüssigkeit um die Gallenblase. Im Falle von Gallenblasensteinen finden sich sonografisch Steinreflexe mit Schallschatten und das Rollen der Konkremente bei Bewegung des Patienten ( $\bullet$ Abb. 9). Der Nachweis ist leicht zu führen; der sichere Ausschluss von Steinen ist mit der Sonografie dagegen nicht möglich.

Wie in der Kasuistik („Fallbeispiel - Gallenblasenhydrops“) dargestellt, ist die Umstellung auf die enterale Ernährung eines der immer wiederkehrenden Probleme bei Intensivpatienten. Dabei stellt sich regelmäßig auch die Frage nach Motilitätsstörungen des Gastrointestinaltrakts bzw. im extremen Fall nach einem lleus. Je ausgeprägter die Magen-Darm-Atonie ist, desto leichter lässt sie sich aufgrund des Flüssigkeits-Gewebe-Kontrasts sonografieren. Der Magen stellt sich im Epigastrium dilatiert mit einer festen und flüssigen Phase dar. Ein leerer Magen lässt sich nur schwer sonografisch darstellen.

Zur Durchführung der Magensonografie setzt man den Schallkopf unterhalb des epigastrischen Winkels für einen Oberbauchlängsschnitt auf. Die Markierung zeigt dabei nach kranial. Für eine standardisierte Darstellung des Magenantrums muss der Schallkopf so weit verschoben werden, dass am unteren Bildrand die V. mesenterica superior und die Aorta zu sehen sind. Diese Darstellung wird als Aortomesenterialebene bezeichnet und erlaubt vergleichbare Messungen des Magenantrumquerschnitts ( Abb. 10).

Auch bei einem Ileus basiert dessen sonografische Darstellung auf einem guten Gewebe-Flüssigkeits-Kontrast. Dabei rückt zusätzlich noch die Beschaffenheit der Darmwände in den Vordergrund. So kann man bei einem Dünndarmileus das typische Strickleiterphänomen beschreiben ( $\triangleright$ Abb. 11). Dieses entsteht durch Auftreiben der Dünndarmfaltierung mit Flüssigkeit. Bei einem Dickdarmileus hingegen sind die Haustren verstrichen und die Darmwand stellt sich glatt und homogen dar. Im Falle eines mechanischen Ileus ist Peristaltik sichtbar; diese fehlt bei der Paralyse. Je ausgeprägter der lleus ist, desto leichter gelingt die sonografische Darstellung.

Es empfiehlt sich, den Ultraschallkopf analog zur abdominellen Auskultation statt des Stethoskops zu benutzen. Dabei werden alle 4 Quadranten systematisch untersucht. Man beginnt am Punctum maximum des Schmerzes. Wir empfehlen für einen pragmatischen Zugang alle 4 Quadranten zunächst in der Transversalebene zu untersuchen. Dabei zeigt die Markierung des Schallkopfes zur rechten Patientenseite. 

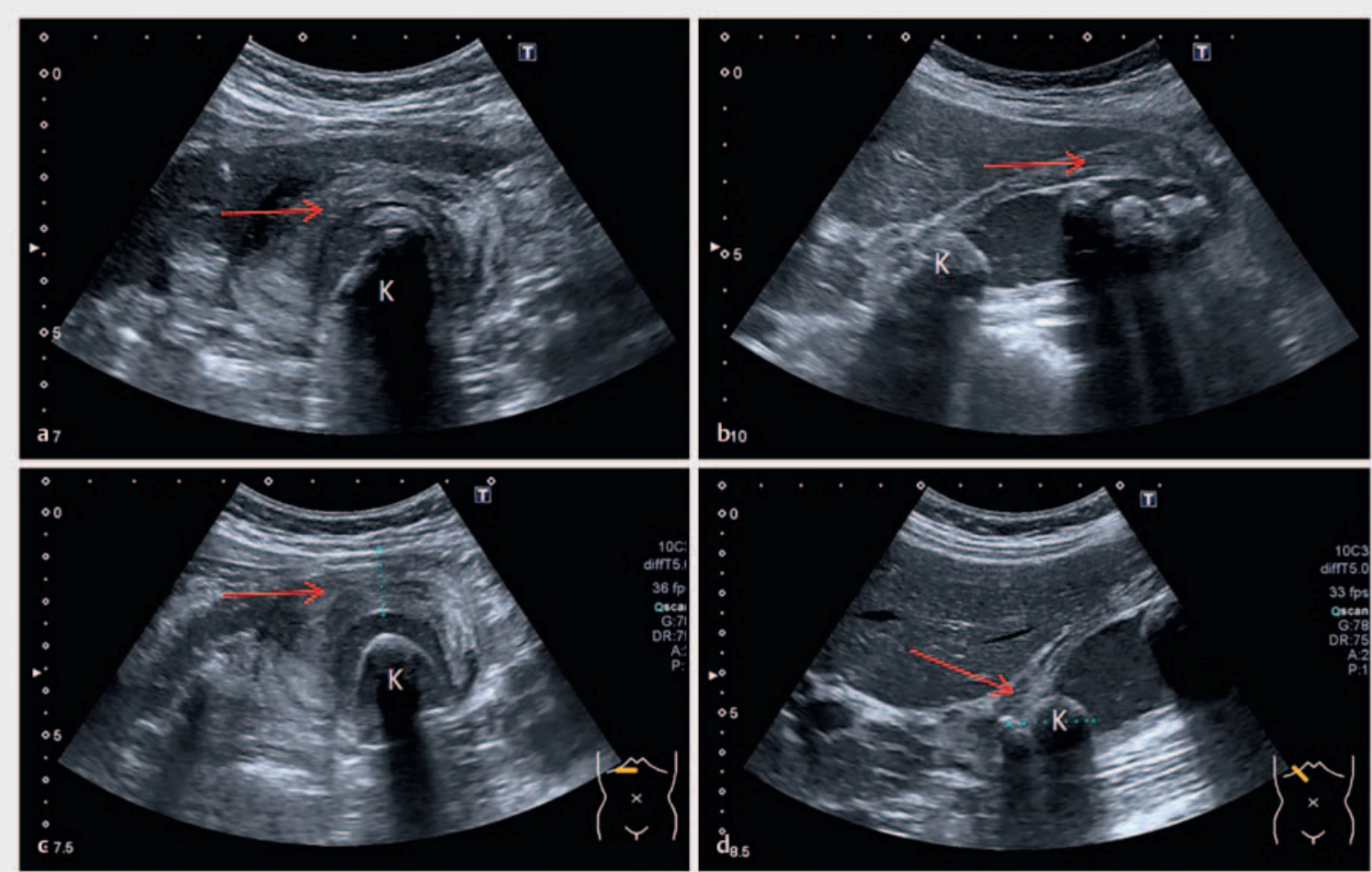

- Abb.9 Nekrotisierende Cholezystitis mit massiver Wandverdickung und Auflösung der Schichtung (Pfeil), infundibuläre Konkrementobstruktion (K). a Subkostaler Schrägschnitt. b Verlängerter Interkostalschnitt. c Subkostaler Schrägschnitt. d Verlängerter Interkostalschnitt. Quelle: Schreiber M, Greim CA. Akutsonografie in der Intensivmedizin: Abdomen. In: Eckart J, Forst H, Briegel J, Hrsg. Intensivmedizin. Kapitel XV - 4.4. Landsberg: ecomed Medizin; 2018: 8.

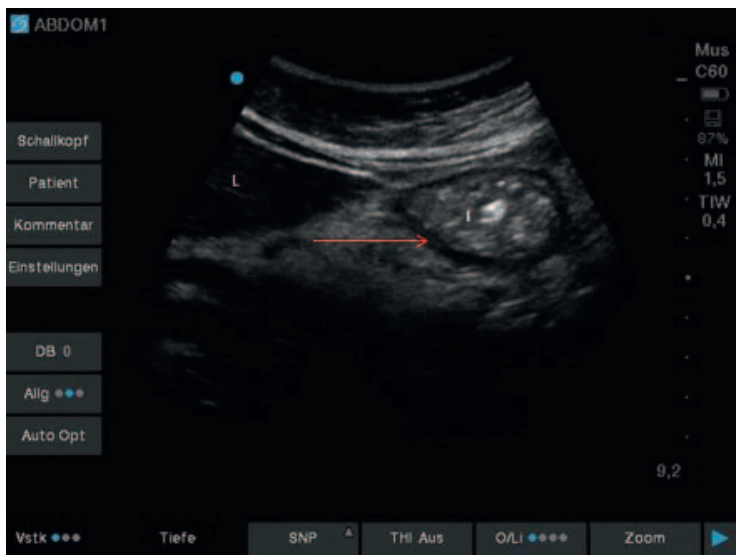

- Abb. 10 Gefüllter Magen im Oberbauchquerschnitt: Magenwand (Pfeil), Mageninhalt (I), Leber (L).

\section{Merke}

Akalkulöse Cholezystitis und Magen-Darm-Atonie sind wichtige sonografische Diagnosen. Bei passenden klinischen Zeichen kann sich hinter dem sonografischen Bild des lleus eine Darmischämie verbergen.

In den ersten Stunden nach Beginn der Symptomatik ist die Sonografie der Röntgendiagnostik überlegen [18-

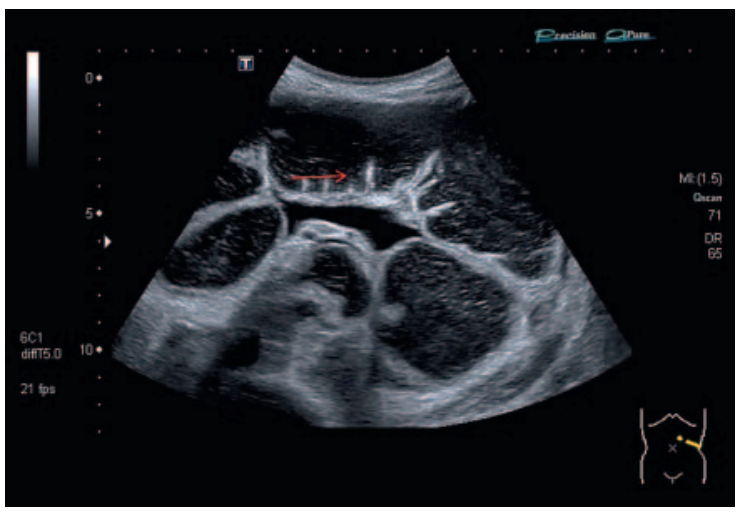

- Abb.11 Dünndarmileus mit Strickleiterphänomen (Pfeil).

20]. Somit hat das Beherrschen der Untersuchungen hohe notfall- und intensivmedizinische Relevanz.

Störungen der Nierenfunktion und der Harnableitung

Die akute Nierenschädigung ist eine der häufigsten Organinsuffizienzen auf der Intensivstation, vor allem im Rahmen einer Sepsis. Von den geläufigen Ursachen, die in prärenal (60\%), intrarenal (35\%) und postrenal (5\%) unterteilt werden, können Letztere durch POC-Sonogra- 
fie sehr einfach detektiert werden. Die postrenale Nierenschädigung tritt verhältnismäßig selten auf, geht bei längerem Bestehen nicht selten in ein intrarenales Nierenversagen über und ist häufig Auslöser für eine Urosepsis. Ursachen sind vor allem Obstruktionen durch Steine, Tumoren und Blutkoagel. Die sonografischen Schnittführungen für die Nieren und die Harnblase sind bereits im Rahmen der FAST-Darstellung zur Anwendung gekommen und können somit leicht übertragen werden.

\section{FALLBEISPIEL}

\section{Urosepsis}

Abends gegen 20:00 Uhr wird eine 74-jährige Patientin mit reduziertem Allgemeinzustand und V. a. septische Pneumonie intubiert und beatmet auf die ITS übernommen. Sie befindet sich im septischen Schock mit akutem Nierenversagen bei hochdosierter Katecholamintherapie. Das initiale Röntgenbild vom Thorax zeigt unerwartet keine verdächtigen Infiltrate. Unter Breitbandantibiotikagabe und Ausgleich des Volumendefizits lässt die Patientin sich hämodynamisch stabilisieren. Da laut Pflegeüberleitung seit einigen Tagen zunehmende Bauchschmerzen bestanden hatten, führt der Intensivmediziner eine orientierende Abdomensonografie zur Fokussuche durch. Dabei gelingt der Nachweis einer linksseitigen Harnstauungsniere, die sofort urologisch therapiert wird. Das verursachende Harnleiterkonkrement wird endoskopisch geborgen und der Urosepsisfokus kausal beseitigt. Trotz zeitnaher therapeutischer Maßnahmen entwickelt die Patientin unter der Sepsis ein anurisches Nierenversagen und muss einige Tage dialysiert werden. In dieser Zeit wird der Blasendauerkatheter entfernt und es erfolgen tägliche Blasensonografien zur Beurteilung der Füllung. Am 10. Tag nach Aufnahme gelingt der Nachweis von Urin. Die Patientin ist inzwischen extubiert und in deutlich gebessertem klinischem Zustand. Es erfolgt die Anlage eines Blasendauerkatheters, der ab diesem Zeitpunkt - im Rahmen der polyurischen Erholung - Urin fördert. Auch die Retentionsparameter normalisieren sich.

Die Anurie stellt den Intensivmediziner regelmäßig vor die Frage eines postrenalen Nierenversagens. Dabei gilt die Notfallsonografie als Mittel der Wahl zur Diagno-

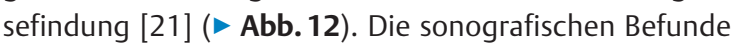
einer Urostase im Nierenbecken werden in 4 Stadien der Hydronephrose eingeteilt (s. „Definition - Hydronephrose-Grade“).

\section{DEFINITION}

\section{Hydronephrosegrade}

- Grad I: echofreie Erweiterung des Nierenbeckens ohne Erweiterung der Kelche - keine Vergrößerung der Niere

- Grad II: echofreie Erweiterung des Nierenbeckens ohne Erweiterung der Kelche - mit Vergrößerung der Niere

- Grad III: echofreie Erweiterung des Nierenbeckens ohne Erweiterung der Kelche - mit Vergrößerung der Niere und Verkleinerung des Nierenparenchyms

- Grad IV: echofreie Erweiterung des Nierenbeckens und Erweiterung der Kelche, wobei die Grenzen von beiden aufgehoben sind

Die genaue Differenzialdiagnostik und die Gradeinteilung obliegen dem Urologen bzw. Radiologen. Die häufigsten Stauungsursachen sind Steine und Blutkoagel. Beide verbergen sich manchmal der direkten Sicht (u. a. aufgrund der Steinart). Dann kann man nur die indirekten sonografischen Zeichen, eine proximale Nieren- und Ureterstauung und den distalen Ureterkollaps, erkennen.

Betont sei nochmals, dass die durchgeführte Notfallsonografie durch den Intensivmediziner die Zeit bis zur urologischen oder nephrologischen Diagnostik verringern, diese aber nicht ersetzen kann.

Merke

Anurie ist ein Symptom des akuten Nierenversagens, dessen Ursache bei postrenalem Abflusshindernis mit der Sonografie schnell eingegrenzt werden kann.

Weniger im intensivmedizinischen Notfall als im Verlauf bietet die Sonografie die Möglichkeit, durch tägliches Screening frühzeitig die renale Erholung und das Wiedereinsetzen der Urinproduktion zu erkennen. Damit lässt sich die Liegedauer des Blasendauerkatheters - als potenzielle Infektionsquelle - während langer anurischer Phasen verkürzen (s. „Fallbeispiel - Urosepsis“).

Auch im Aufwachraum oder auf der peripheren Station kann der Akuteinsatz der Sonografie zur Abklärung einer Anurie sinnvoll sein und schnell ein Ergebnis liefern: Eine übervolle Harnblase als Zeichen einer postvesikalen Abflussstörung, z. B. nach Spinalanästhesie oder bei hohem Opioidbedarf, kann mithilfe der oben aufgeführten FASTPosition 5 verhältnismäßig einfach dargestellt werden. 

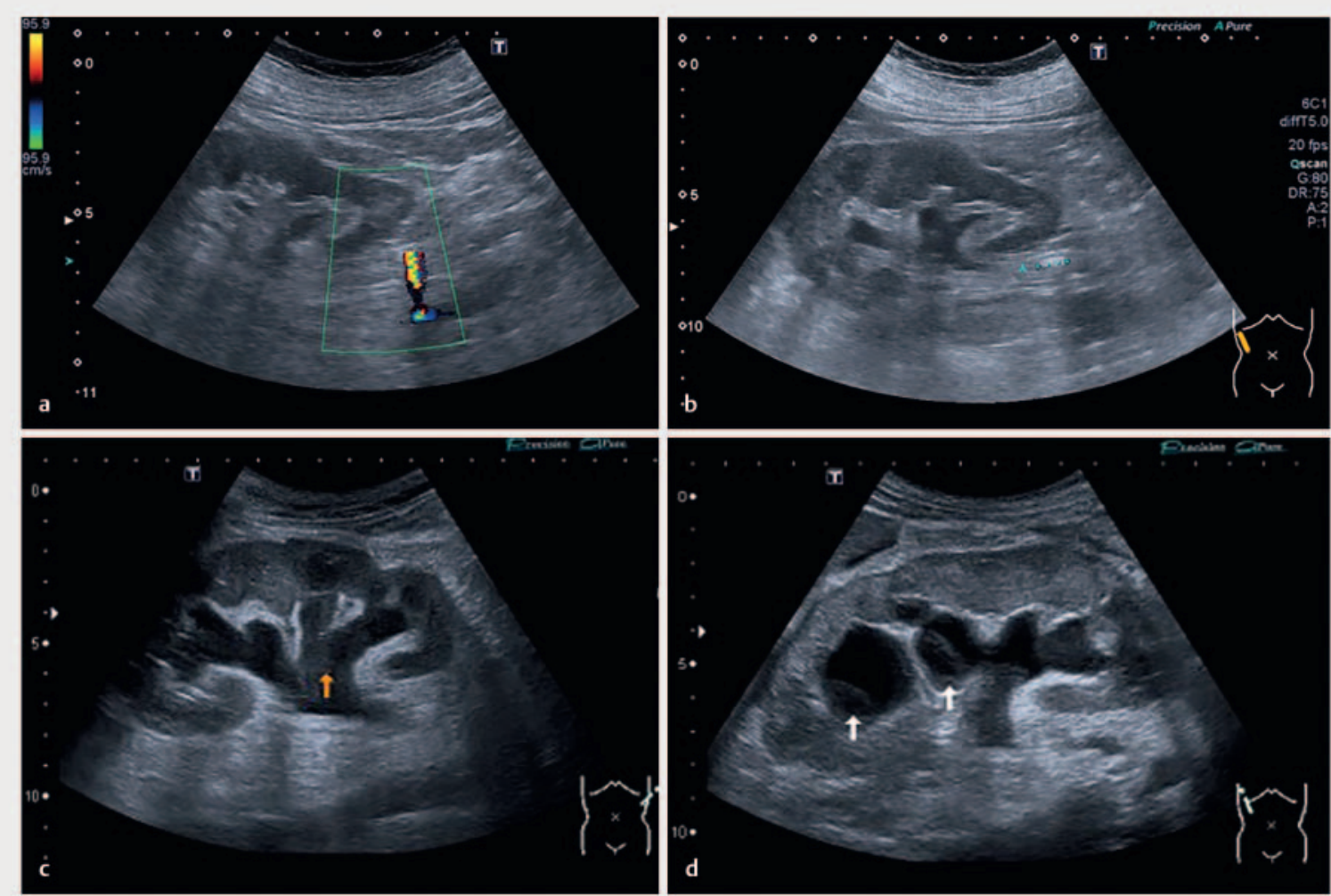

- Abb. 12 Notfallsonografie bei Störungen der Harnableitung. a Kleines Ureterkonkrement mit Twinkling-Artefakt zu erkennen. b Ureterkonkrement und Stauung I $^{\circ}$ des Nierenbeckenkelchsystems (NBKS). c Nierenbeckenstauung, NBKS-Stauung II-III . d Spiegel im Nierenbecken bei Pyonephrose. Quelle: Schreiber M, Greim CA. Akutsonografie in der Intensivmedizin: Abdomen. In: Eckart J, Forst H, Briegel J, Hrsg. Intensivmedizin. Kapitel XV - 4.4. Landsberg: ecomed Medizin; 2018: 10.

\section{Fazit und Ausblick}

Die abdominelle Notfallsonografie adressiert zahlreiche Fragestellungen, die sich häufig finden

- bei Patienten auf den Intensivstationen,

- in der präklinischen Notfallmedizin,

- in der Notaufnahme,

- oft auch in Spät-, Nacht- oder Bereitschaftsdiensten,

- aber auch im postoperativen Betreuungsbereich.

Aus den sonografisch gestellten, schnell erhobenen und richtigen Diagnosen ergeben sich für den Behandlungsverlauf regelmäßig positive Therapieentscheidungen.

Derzeit bieten die sonografischen Ausbildungskurse in der Anästhesiologie und Intensivmedizin keine ausreichende Möglichkeit, um sich für eine definitive sonografische Diagnostik des Abdomens zu qualifizieren. Es bleibt somit beim Ansatz, mit einer fokussierten Untersuchung den Weg für eine weiterführende Untersuchung zu öffnen und die Verdachtsdiagnose durch fachärztliche Konsile und Expertise der zuständigen Fachgebiete abzusichern. Doch auch die fokussierte Abdomensonografie bedarf noch weiterer Qualifizierungsoptionen, die entwi- ckelt werden müssen und künftig Teil der Seminarreihe „Anästhesie Fokussierte Sonografie“ sein könnten.

Viele der dargestellten sonografischen Einstellungen sind in mehrstündigen Kursen leicht zu erlernen und lassen sich als Ausgangspunkt für eine weitergehende konsekutive und dezidierte Diagnostik nutzen. Nicht alle gegenwärtig in der Notfall-Abdomensonografie genutzten Techniken und Schnitte konnten im Rahmen des Artikels ausführlich angesprochen werden. Weiterführende Inhalte und Möglichkeiten betreffen hauptsächlich die Sonoanatomie der Abdominalgefäße inkl. Bauchaortenaneurysmata sowie Pathologien des Darms (Appendizitis, Kolitis, Divertikulitis, Perforation mit freier Luft).

Aufgrund der vielen Vorteile und Möglichkeiten der POCSonografie ist im Bereich der Intensiv- und Notfallmedizin zunehmend mit ihrem Einsatz zu rechnen. Perspektivisch sollte jeder Intensiv- und Akutmediziner über ein Mindestmaß von sonografischen Fähigkeiten verfügen. Die Erarbeitung eines intensivmedizinischen Curriculums durch die Fachgesellschaften wird ein folgerichtiger Schritt sein. Damit wird das Erlernen der Notfall-Abdomensonografie systematisiert und zu einer guten Investition für Arzt und Patient. 


\section{KERNAUSSAGEN}

- Das Fehlen fachspezifischer und organbezogener sonografischer Expertise außerhalb des Regeldienstes und in zeitkritischen akutmedizinischen Situationen verleiht der POC-Sonografie für den Notfall- und Intensivmediziner hohen Stellenwert.

- Der Konvexschallkopf ist für die abdominelle Sonografie konzipiert.

- Die FAST-Sonografie dient primär dem Nachweis von freier intraabdomineller Flüssigkeit in hämodynamisch instabilen Situationen. Darüber hinaus kann sie als Einstieg und Orientierungshilfe für die abdominelle Sonografie gelten.

- Störungen der Gallenwege und gastrointestinalen Motilität gehören regelmäßig zu Problemen des Intensivpatienten. Durch wenige sonografische Schnitte und Blickdiagnosen erwachsen relevante therapeutische Konsequenzen. Gefahrenträchtige Transporte zur CT können u. U. vermieden oder zumindest reduziert werden.

- Einschränkungen oder Passagebehinderungen der Urinproduktion sind wichtige Differenzialdiagnosen, vor allem im Rahmen der Sepsis. Zur Ursachendetektion steht die Sonografie einfach und bettseitig zur Verfügung. Zur Abklärung des Harnstaus ist sie bereits in Leitlinien verankert.

- Für den hohen Bedarf an notfall- und intensivmedizinischen Fragestellungen wird ein konkretes Ausbildungscurriculum für abdominelle POC-Sonografie ein folgerichtiger Schritt sein. Jeder Akutmediziner sollte zeitgemäß über ein Mindestmaß an sonografischen Fähigkeiten verfügen.

\section{Wissenschaftlich verantwortlich gemäß Zerti- fizierungsbestimmungen}

Wissenschaftlich verantwortlich gemäß Zertifizierungsbestimmungen für diesen Beitrag ist Dr. med. Michael Schreiber, Aschaffenburg.

\section{Interessenkonflikt}

\section{Erklärung zu finanziellen Interessen}

Forschungsförderung erhalten: nein; Honorar/geldwerten Vorteil für Referententätigkeit erhalten: nein; Bezahlter Berater/interner Schulungsreferent/Gehaltsempfänger: nein; Patent/Geschäftsanteile/Aktien (Autor/Partner, Ehepartner, Kinder) an Firma: nein.

Erklärung zu nichtfinanziellen Interessen

Die Autorinnen/Autoren geben an, dass kein Interessenkonflikt besteht.

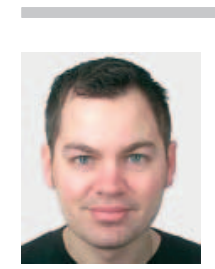

\section{Michael Schreiber}

Dr. med., MHBA, DESA. Facharztausbildung Anästhesiologie am Klinikum Fulda. Zusatzbezeichnungen Notfallmedizin, spezielle Intensivmedizin, Schmerztherapie, Palliativmedizin.

Zuletzt Funktionsoberarzt der Klinik für Anästhesiologie, Intensiv- und Notfallmedizin am Klinikum Fulda. Schwerpunkte: Intensivmedizin, POC-Sonografie. Seit Mitte 2018 Oberarzt an der Klinik für Anästhesiologie und operative Intensivmedizin am Klinikum Aschaffenburg-Alzenau.

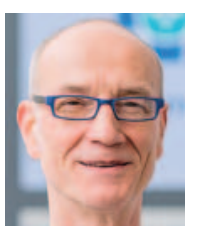

\section{Clemens-Alexander Greim}

Prof. Dr. med., Direktor der Klinik für Anästhesiologie, Intensiv- und Notfallmedizin am Klinikum Fulda. Seit 2011 Leiter des Arbeitskreises Ultraschall der DGAI. Schwerpunkte: klinische Versorgung, perioperative Sonografie, Qualitäts- und Gesundheitsmanagement.

\section{Korrespondenzadresse}

\section{Dr. med. Michael Schreiber, DESA, MHBA}

Klinik für Anästhesiologie und operative Intensivmedizin Klinikum Aschaffenburg-Alzenau

Am Hasenkopf

63739 Aschaffenburg

Michael.Schreiber@klinikum-AB-ALZ.de

\section{Danksagung}

Die Autoren danken Andreas Haars und Nora Stan für ihre Unterstützung bei der Zusammenstellung des Bildmaterials.

\section{Zitierweise für diesen Artikel}

Anästhesiol Intensivmed Notfallmed Schmerzther 2019; 54 : 128-141. doi:10.1055/a-0585-5237

\section{Literatur}

[1] Moore CL, Copel JA. Point-of-care ultrasonography. N Engl J Med 2011; 364: 749-757

[2] American College of Emergency Physicians. Emergency Ultrasound guidelines. Ann Emerg Med 2009; 53: 550-570

[3] DGAI Arbeitskreis Ultraschall. AFS-Seminarreihe (Februar 2012). Im Internet: http://www.ak-ultraschall.dgai.de/informa tionen-und-links/downloads/afs-seminarreihe.html; Stand: 31.10.2018

[4] DEGUM Arbeitskreis Notfallsonographie. Kurse \& Kurscurricula. Im Internet: http://www.degum.de/arbeitskreise/notfallsono graphie/kurse-kurscurricula.html; Stand: 31.10.2018

[5] Dolich MO, McKenney MG, Varela JE et al. 2576 ultrasounds for blunt abdominal trauma. J Trauma 2001; 50: 108-112

[6] Scalea TM, Rodriguez A, Chiu WC et al. Focused assessment with sonography for Trauma (FAST): Results from an international consensus conference. J Trauma 1999; 46: 466-472

[7] Kirkpatrick AW, Sirois M, Laupland KB et al. Hand-held thoracic sonography for detecting post-traumatic pneumothoraces: The 
Extend Focused Assessment with Sonography for Trauma (EFAST). J Trauma 2004; 57: 288-295

[8] Thies K, Gwinnutt C, Driscoll P et al. The European Trauma Course - From concept to course. Resuscitation 2007; 74 135-141

[9] American College of Surgeons' Committee on Trauma; ATLS Subcommittee; International ATLS working group. Advanced trauma life support (ATLS®). 9th ed. J Trauma Acute Care Surg 2012; 74: 1363-1366

[10] Körner M, Krötz MM, Degenhart C et al. Current Role of Emergency US in Patients with Major Trauma. Radiographics 2008; 28: 225-242

[11] Kendall JL, Hoffenberg SR, Smith RS. History of emergency and critical care ultrasound. Crit Care Med 2007; 35: 126-130

[12] Walcher F, Krisching T, Müller MP et al. Accuracy of prehospital focused abdominal sonography for trauma after a 1-day hands-on training course. Emerg Med J 2010; 27: 345-349

[13] Greim CA, Roewer N. Transösophageale Echokardiografie für Intensivmediziner und Anästhesisten. 3. Aufl. Stuttgart: Thieme; 2007

[14] Hahn D, Offermann S, Holmes J. Clinical importance of intraperitoneal fluid in patients with blunt intra-abdominal injury. Am J Emerg Med 2002; 20: 595-600

[15] Rozycki G, Ochsner M, Feliciano D et al. Early detection of hemoperitoneum by ultrasound examination of the right upper quadrant: a multicenter study. J Trauma 1998; 45: $878-883$
[16] Reinhard K, Brunkhorst FM, Bone HG et al. Prävention, Diagnose, Therapie und Nachsorge der Sepsis. Anästhesist 2010; 59: 347-370

[17] Markose J, Büter M. Akute Galle - Eine Herausforderung auf der Intensivstation. Viszeralchirurgie 2007; 42: 22-26

[18] Guttman J, Stone MB, Kimberly HH et al. Point-of-care ultrasonography for the diagnosis of small bowel obstruction in the emergency department. CJEM 2015; 17: 206-209

[19] Jang TB, Schindler D, Kaji AH. Bedside ultrasonography for the detection of small bowel obstruction in the emergency department. Emerg Med J 2011; 28: 676-678

[20] Taylor MR, Lalani N. Adult small bowel obstruction. Acad Emerg Med 2013; 20: 528-544

[21] Stoller M, Aubin C, Bailitz J et al. A randomized trial of ultrasound versus computed tomography for imaging patients with suspected nephrolithiasis'. Annual Meeting of the American Urological Association, Orlando, FL, May 2014. J Urol 2014; 191 (Suppl. 4): e51-e52

Bibliografie

DOI https://doi.org/10.1055/a-1007-9241

Dtsch Med Wochenschr 2020; 145: 104-117

(c) Georg Thieme Verlag KG, Stuttgart · New York

ISSN 0012-0472 


\section{Punkte sammeln auf CMEthieme.de}

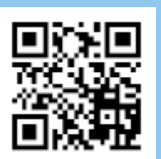

Diese Fortbildungseinheit ist in der Regel 12 Monate online für die Teilnahme verfügbar.

Den genauen Einsendeschluss finden Sie unter https://eref.thieme.de/CXDTH4D.

Sollten Sie Fragen zur Online-Teilnahme haben, finden Sie unter https://cme.thieme.de/hilfe

eine ausführliche Anleitung. Wir wünschen viel Erfolg beim Beantworten

der Fragen!

Unter https://eref.thieme.de/CXDTH4D oder über den QR-Code kommen Sie direkt zur Startseite des Wissenstests.

VNR 2760512020158721221

\section{Frage 1}

Welche Aussage ist richtig?

A Nur ausgewiesene Sonografieexperten können wirklich therapierelevante Aussagen treffen.

B Ohne detaillierte Messungen sind sonografische Befunde nicht verwertbar.

C Die POC-Sonografie ermöglicht, regelmäßig in zeitkritischen akutmedizinischen Situationen therapierelevante Befunde zu erheben.

D POC-Sonografie lässt sich nur aufgrund jahrzehntelangen Trainings sicher durchführen.

E POC-Sonografie ist dank ständiger 24-Stunden-Präsenz organspezifischer Ultraschallexperten entbehrlich.

\section{Frage 2}

Welche Aussage ist richtig?

A Sonografie kommt in der Notfall- und Intensivmedizin extrem selten zum Einsatz.

B Röntgenuntersuchungen sind in sämtlichen Belangen der Sonografie überlegen.

C Bei negativem FAST-Befund, massiver Kinetik und instabilem Kreislauf muss zwingend eine $\mathrm{CT}$ erfolgen.

D Das Einbinden sonografischer Untersuchungen in Algorithmen behindert den reibungslosen Ablauf und hat keine Konsequenz.

E Außer im Schockraum hat das FAST-Konzept keinen weiteren Stellenwert.

\section{Frage 3}

Welche FAST-Positionen sind für die Diagnostik eines Pleuraergusses am besten geeignet?
A FAST-Position 1 und 2.
B FAST-Position 1 und 3.
C FAST-Position 1 und 4.
D FAST-Position 1 und 5.
E FAST-Position 1 und 6 .

\section{Frage 4}

Welche der FAST-Positionen wird auch als „subkostaler Vierkammerblick" bezeichnet?
A FAST-Position 1.
B FAST-Position 3.
C FAST-Position 5.
D FAST-Position 6.
E FAST-Position 7.

\section{Frage 5}

Die FAST-Position 4 zeigt den sog. Koller-Pouch an. Um welchen Raum handelt es sich dabei?

A Um den Raum zwischen oberem Nierenpol und Milz.

B Um den Raum zwischen oberem Nierenpol und Leber.

C Um die Pleurahöhle.

D Um den Raum zwischen Herz und Perikard.

E Um den retrouterinen Raum.

\section{Frage 6}

Welche Aussage ist falsch?

A Ein leerer Magen lässt sich nicht gut sonografisch darstellen.

B Peristaltik lässt sich in keinem Falle sonografisch darstellen.

C Grundlage der Sonografierbarkeit des Magen-Darm-Traktes ist der Gewebe-Flüssigkeits-Kontrast bei den meisten Pathologien.

D Ein sonografisches Charakteristikum des Dünndarmilieus ist das Strickleiterphänomen.

E Hinter einem lleus kann sich eine Ischämie verbergen.

\section{Frage 7}

Welche Aussage ist richtig? Die Aortomesenterialebene ...

A ist die standardisierte Darstellung der Gallenblase.

B ist die standardisierte Darstellung des Magenantrums.

C wird dargestellt, wenn am oberen Bildrand die Aorta und die A. mesenterica zu sehen sind.

D erlaubt vergleichbare Messungen des Aortenquerschnitts.

E ist bei Gallensteinen nur sehr schwierig darstellbar.

- Weitere Fragen auf der folgenden Seite... 


\section{Punkte sammeln auf CME.thieme.de}

\section{Frage 8}

Welche Aussage ist falsch?

A Die CT ist Mittel der ersten Wahl zur Diagnose einer Gallenblasenentzündung.

B Bei sedierten und beatmeten Intensivpatienten kann die klinische Untersuchung des Abdomens erschwert oder nicht möglich sein.

C Störungen des Magen-Darm-Traktes und der Gallenwege sind häufige Probleme auf einer Intensivstation.

D Sonografie kann helfen, Röntgenuntersuchungen zu reduzieren und potenziell gefährliche Intensivtransporte zur CT zu vermeiden.

E Das Vorhandensein von Gallensteinen kann in der Sonografie nicht zu $100 \%$ ausgeschlossen werden.

\section{Frage 9}

Welche Aussage ist falsch?

A Die Ursachen einer akuten Nierenschädigung auf der Intensivstation werden unterteilt in prärenal $(60 \%)$, intrarenal $(35 \%)$, postrenal (5\%).

B Die sonografischen Befunde einer Urostase im Nierenbecken werden in 4 Stadien der Hydronephrose eingeteilt.

C Die postrenale Nierenschädigung ist häufig Auslöser einer Urosepsis.

D Die prärenalen Ursachen einer Nierenschädigung können sehr einfach durch POC-Sonografie diagnostiziert werden.

E Die Ursachen einer postrenalen Nierenschädigung sind vor allem Obstruktionen durch Steine, Tumoren o. Ä.

\section{Frage 10}

Welche Aussage ist richtig?

A Die Sonografie des Abdomens ist ein Bereich, der auch zukünftig ausschließlich in der Hand von Experten bleiben wird.

B Differenzierte Messungen und detailgetreue Schnittführungen machen aufgrund der POC-Sonografie zukünftig keinen Sinn mehr.

C POC-Sonografie soll die differenzierte Organdiagnostik nicht ersetzen, sondern genau umschriebene klinische Fragestellungen in zeitkritischen Situationen beantworten.

D POC-Sonografie kostet nur Zeit und hat keine therapeutischen Konsequenzen.

E Auch in Zukunft besteht keine Veranlassung für Intensiv- und Notfallmediziner, POC-Sonografie zu erlernen. 